\title{
Acute kidney Injury in children: etiologies and results
}

\section{Seba Atmane}

Department of Nephrology, University Hospital Tizi Ouzou, ALGERIA

*Corresponding Author: Seba Atmane, Department of Nephrology, University Hospital Tizi Ouzou, ALGERIA.

Received date: May 26 2020; Accepted date: June 18, 2020; published date: June 23, 2020

Citation: Seba Atmane, Acute kidney Injury in children: etiologies and results, J Clinical Case Reports and Studies. 1(2); DOI: 10.31579/2690-

8808/008

Copyright: Copyright (C) 2020 Seba Atmane. This is an open access article distributed under the Creative Commons Attribution License, which permits unrestricted use, distribution, and reproduction in any medium, provided the original work is properly cited.

\section{Abstract}

The aim of the study is to show the etiologies and the follow-up of our AKI cases. This was conducted in our hospital, between 2015 and 2018. during this period we included 26 children with AKI (64\% femals) with a median age of 7 years (range 40 days to 15 years). In the majority of the cases revealed by digestive signs and that related to the etiology of AKI (Hemolytic Uremic Syndrome post diarrhea). In our study, $44 \%$ of the patients have thrombocytopenia associated with AKI. The etiology of AKI is : Nephropathy glomerular in $37 \%$ hemolytic and uremic syndrom in 54\% and obstructive nephropathy in $9 \%$. Patients survived in $92 \%$ of the cases and $58 \%$ of them have recovered normal kidney function, $7 \%$ of death. Peritoneal dialysis is the most commonly used emergency treatment for AKI in children at a frequency of $37 \%$., hemodialysis was used less.

Key words: AKI; hemolytic-uremic syndrome

\section{Introduction}

Acute kidney Injury (AKI) is characterized by a reversible increase in the blood concentration of creatinine and nitrogenous waste products and by the inability of the kidney to regulate fluid and electrolyte homeostasis appropriately [1]. There are many causes of AKI.

Some causes of AKI, such as rapidly progressive glomerulonephritis, may present as AKI but rapidly evolve into chronic kidney disease (CKD). Several renal diseases, such as the hemolytic-uremic syndrome (HUS) and obstructive uropathy with associated renal dysplasia, may present as AKI with improvement of renal function to normal or near-normal levels, but the child's renal function may slowly deteriorate, leading to CKD several months to years later.

\section{Materials and methods}

It is a retrospective, conducted between 2015 and 2018, the patients were selected from the hospitalization register. We included all children with AKI during this time period. Data analysis was based on patient records.
We have studied the following: - Age, sex, etiological diagnosis, evolution.

\section{Results}

Over the four years, 26 patients (34\% male) with a median age of 7 years (range 40 days to 15 years) were managed. The most affected age group in our study is [12-16] with a frequency of $36 \%$.

The circumstances of discovery in the majority revealed by digestive signs and that related to the etiology of AKI (HUS post diarrhea). In our study, $44 \%$ of the patients have thrombocytopenia associated with AKI; and this is due to the origin of this syndrome (Atypical HUS). The etiology of AKI is: Nephropathy glomerular in 37\%, HUS in 54\% and obstructive nephropathy in 9\%. Patient follow-up showed, survived in 92 $\%$ of the cases and $58 \%$ of them have recovered normal kidney function, $7 \%$ of death. Peritoneal dialysis is the most commonly used emergency treatment for AKI in children at a frequency of $37 \%$.

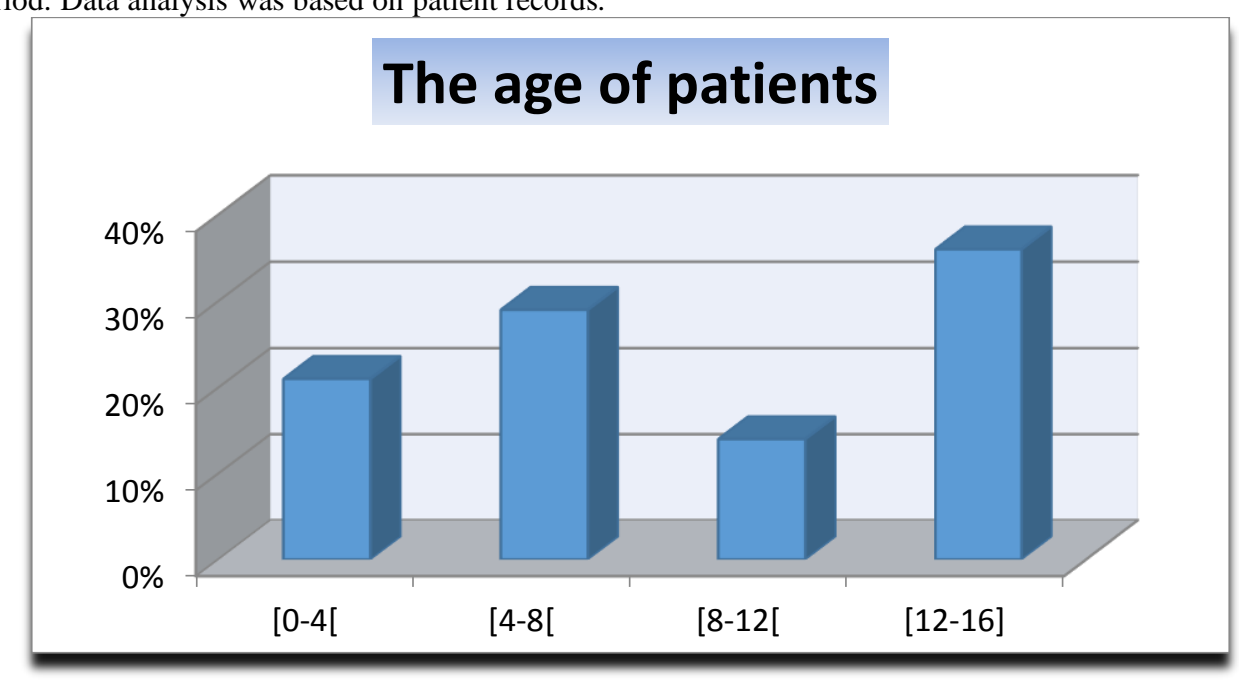



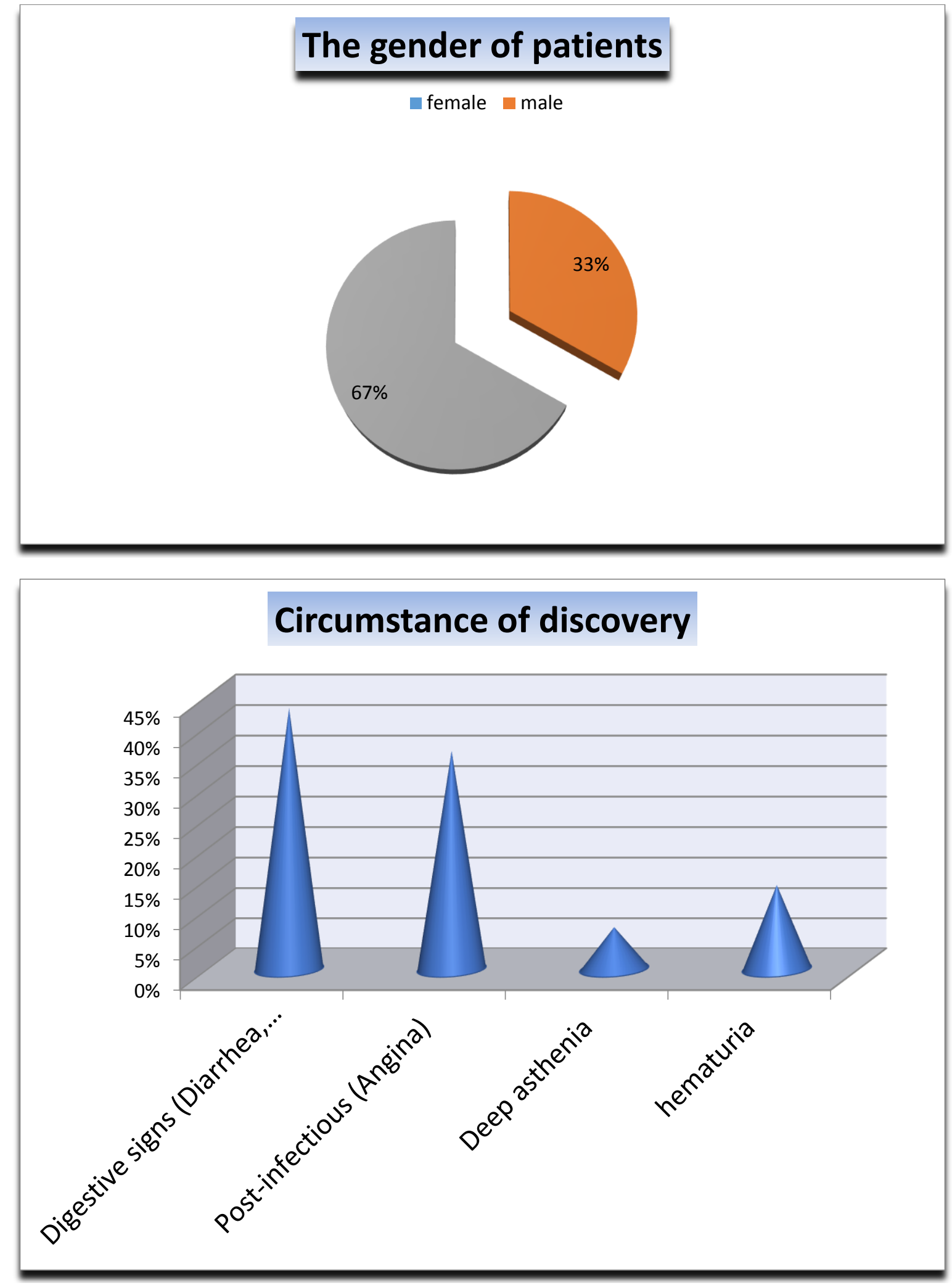

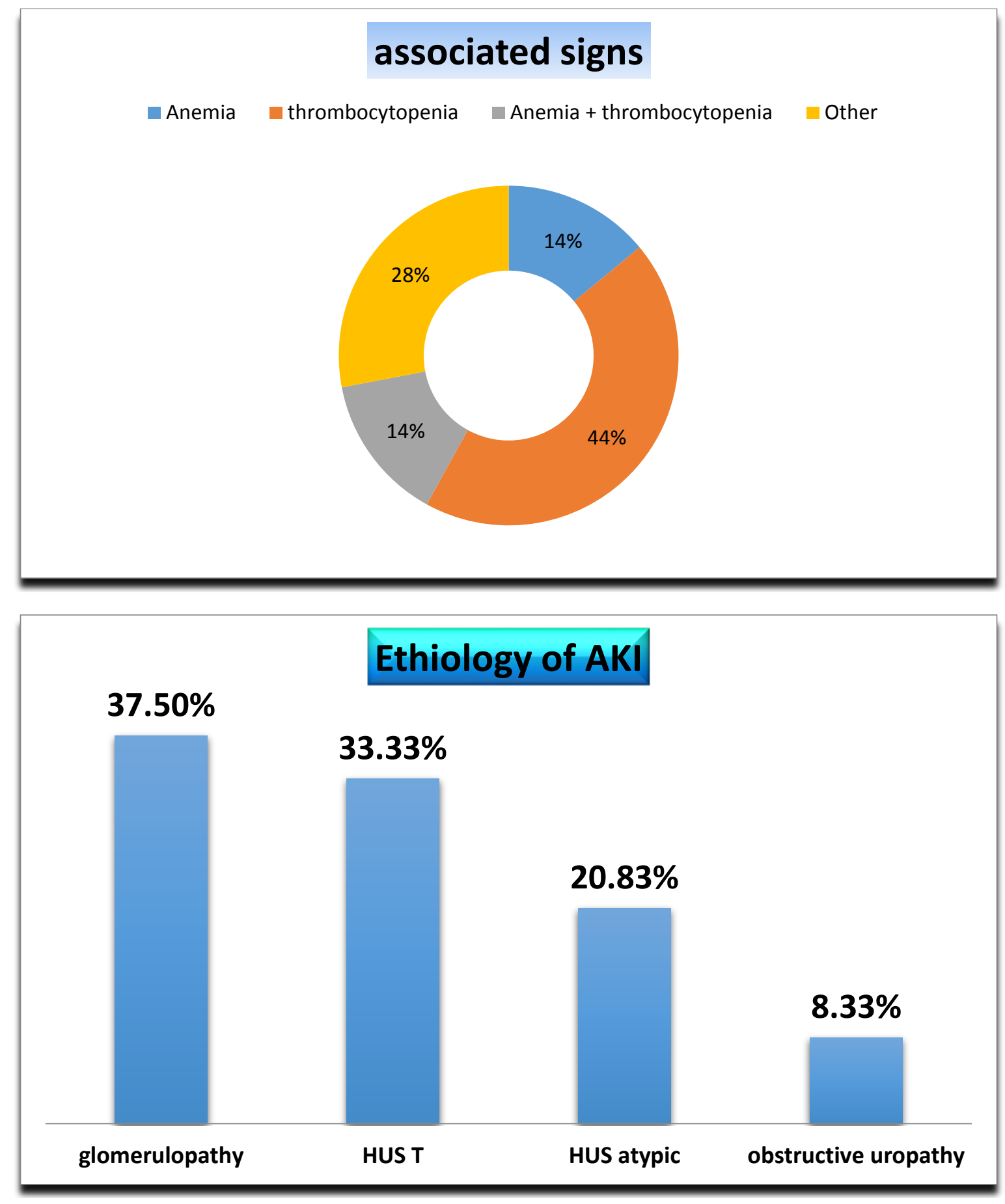

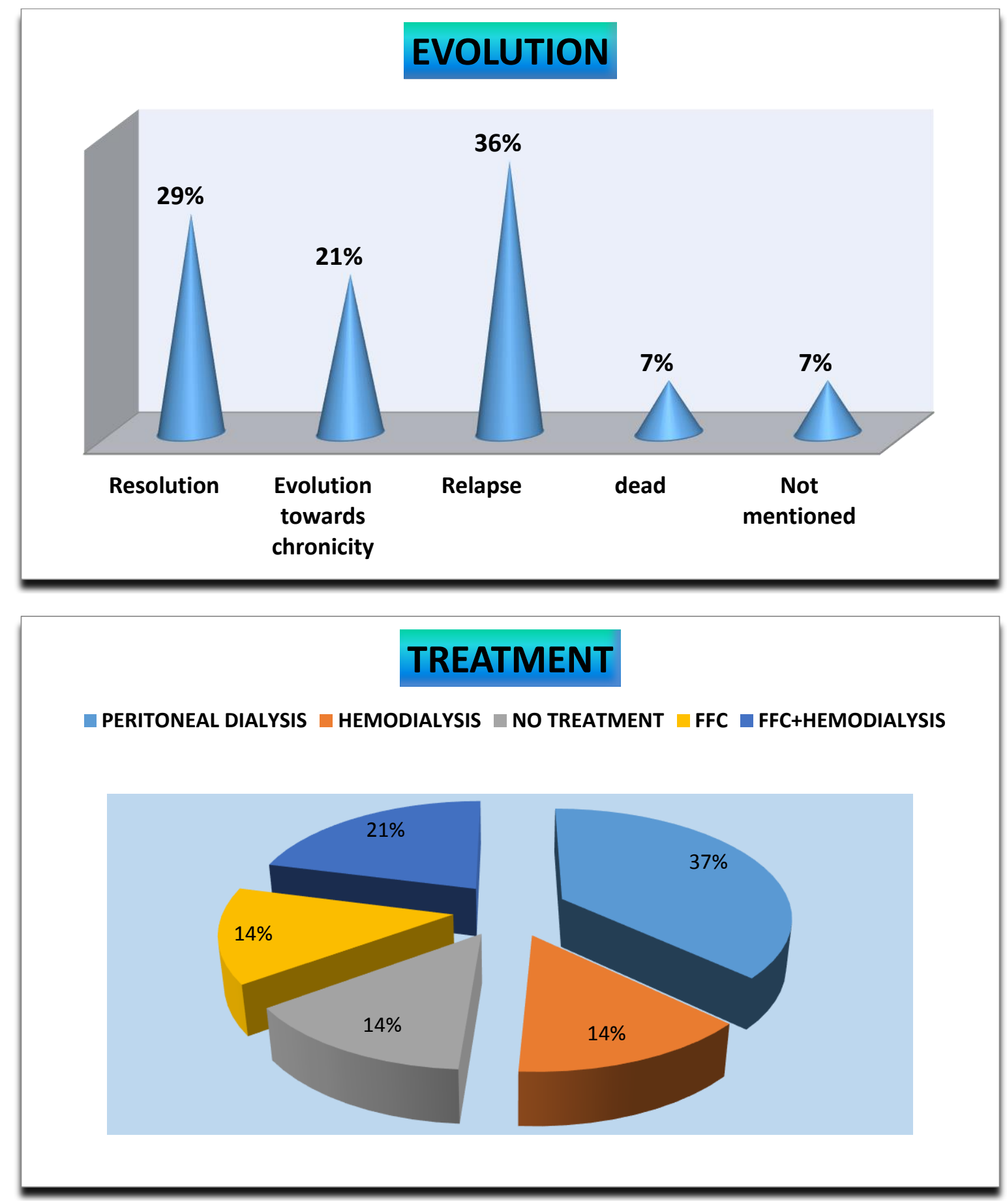

\section{Discussion}

The prevalence of AKI is variously appreciated in the literature. Most of the data come from intensive care units, post-surgery resuscitation, neonatology or nephrology where the type of recruitment of children is different from one service to another [2].The average age of the children in our work was 7 years. In 2001, in a pediatric ward in Alabama (USA) Bunchmann et al. Reported an average age of 6.16 years. Most authors agree that the etiologies of AKI in children vary by age. Despite variations, two causes dominate, AKI secondary to renal hypoperfusion and Hemolytic Uremic Syndrome (HUS) $[1,2,3,4]$. The first cause occurs at any age, while HUS, in typical form, is unique to infants and young children under three years of age, in our experience. Five patients $(36 \%)$ were feverish at admission. This testifies to the responsibility of infections in the genesis of nephropathies.

The severity of the renal involvement seems to affect the renal functional prognosis. Indeed, elevated serum creatinine and blood urea are predictive of renal failure. The risk of hyperkalemia exists as soon as an AKI sets up. Any serum potassium greater than $6 \mathrm{mmol} / 1$ is at risk for severe heart rythm disorders. Anemia was found in two patients (14\%). 
Etiologically, the most common cause in our study was HUS with a frequency of 54\%. Post infectious glomerulonephritis was present in 36\% of cases in our study. Its frequency is appreciated in the USA with $12.6 \%$ cases $i$. The acute glomerulonephritis in a tropical environment is poststreptococcal and is often associated with an AKI. Recovery is usually complete and does not require biopsy. However, biopsy may be indicated in cases of rapidly progressive glomerulonephritis. In other studies, organic AKI of toxic origin is described. This nephrotoxicity is observed in oncology in children treated with Cisplatin or Methotrexate. Causes of post-renal AKI in children are uncommon.Therapeutically, peritoneal dialysis practiced in the majority of our cases [5], hemodialysis was prescribed in $14 \%$ of our patients. Currently, because of the effectiveness of extra-renal cleansing techniques, mortality is most often linked to the etiological context and not to the consequences of AKI. On the follow up, 04 patients $(29 \%)$ had evolved favorably, with a complete recovery of renal function. An AKI poorly supported, or the installation of a bilateral cortical necrosis immediately evolve the patient to the chronicity noted in $03 \%$ of our patients.

\section{Conclusion:}

The occurrence of acute kidney Injury in child is a fairly common situation that requires a diagnostic approach: recognize etiology (functional, obstructive or organic AKI) and clinical and biological disturbances to implement quickly route the adapted therapy. It is also important to emphasize the prevention of kidney failure in risk situations.
The long-term prognosis of acute kidney Injury in child depends on their etiology.

\section{Conflicts of Interest}

The authors declare that there are no conflicts of interest.

\section{References:}

1. Acute kidney injury in children Sharon Phillips AndreolAcute kidney injury in children Sharon Phillips Andreol . Pediatr Nephrol (2009) 24:253-263

2. A Retrospective Study of Acute Renal Failure in Children: Its Incidence, Etiology, Complications and Prognosis ,Kadar Ismail Hassan , Jama Hodan M , Chunfu Li . Cureus 9(5): e1274.

3. Pediatric Acute Kidney Injury: Different From Acute Renal Failure But How And Why ,Prasad Devarajan, MD . Curr Pediatr Rep. Author manuscript; available in PMC 2014 March 01.

4. Acute kidney injury: A pediatric experience over 10 years at a tertiary care center,Alaleh Gheissari* , Pardis Mehrasa, Alireza Merrikhi, Yahya Madihi. J Nephropathology. kidney injury in children.

5. Renal replacement therapy for acute renal failure in children: European Guidelines, Vladimirs Strazdins · Alan R. Watson · Ben Harvey. Pediatr Nephrol (2004) 19:199-207

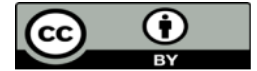

This work is licensed under Creative Commons Attribution 4.0 License

To Submit Your Article Click Here: Submit Manuscript

DOI: $10.31579 / 2690-8808 / 008$
Ready to submit your research? Choose Auctores and benefit from:

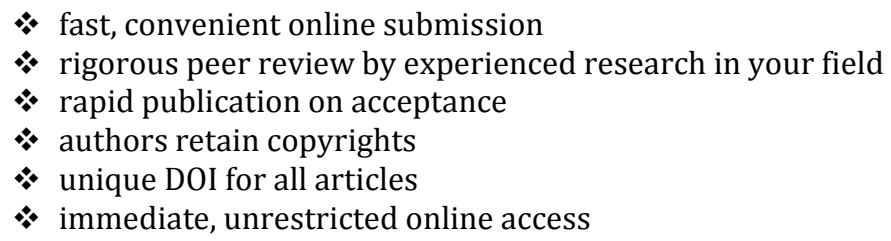

At Auctores, research is always in progress.

Learn more www.auctoresonline.org/journals/journal-of-clinical-casereports-and-studies 